\title{
Technology Challenges for Deep-Throttle Cryogenic Engines for Space Exploration
}

\author{
Kendall K. Brown and Karl W. Nelson \\ Liquid Engine \& MPS Systems Branch, NASA Marshall Space Flight Center, Huntsville, AL 35812 \\ (256) 544-5938, fax (256) 544-5876, kendall.k.brown@nasa.gov
}

\begin{abstract}
Historically, cryogenic rocket engines have not been used for in-space applications due to their additional complexity, the mission need for high reliability, and the challenges of propellant boil-off. While the mission and vehicle architectures are not yet defined for the lunar and Martian robotic and human exploration objectives, cryogenic rocket engines offer the potential for higher performance and greater architecture/mission flexibility. In-situ cryogenic propellant production could enable a more robust exploration program by significantly reducing the propellant mass delivered to low earth orbit, thus warranting the evaluation of cryogenic rocket engines versus the hypergolic bipropellant engines used in the Apollo program. A multi-use engine, one which can provide the functionality that separate engines provided in the Apollo mission architecture, is desirable for lunar and Mars exploration missions because it increases overall architecture effectiveness through commonality and modularity. The engine requirement derivation process must address each unique mission application and each unique phase within each mission. The resulting requirements, such as thrust level, performance, packaging, burn duration, number of operations; required impulses for each trajectory phase; operation after extended space or surface exposure; availability for inspection and maintenance; throttle range for planetary descent, ascent, acceleration limits and many more must be addressed. Within engine system studies, the system and component technology, capability, and risks must be evaluated and a balance between the appropriate amount of technology-push and technology-pull must be addressed. This paper will summarize many of the key technology challenges associated with using high-performance cryogenic liquid propellant rocket engine systems and components in the exploration program architectures. The paper is divided into two areas. The first area describes how the mission requirements affect the engine system requirements and create system level technology challenges. An engine system architecture for multiple applications or a family of engines based upon a set of core technologies, design, and fabrication approaches may reduce overall programmatic cost and risk. The engine system discussion will also address the characterization of engine cycle figures of merit, configurations, and design approaches for some in-space vehicle alternatives under consideration. The second area evaluates the component-level technology challenges induced from the system requirements. Component technology issues are discussed addressing injector, thrust chamber, ignition system, turbopump assembly, and valve design for the challenging requirements of high reliability, robustness, fault tolerance, deep throttling, reasonable performance (with respect to weight and specific impulse).
\end{abstract}

\section{INTRODUCTION}

This paper addresses the application of deep-throttling, multi-use, in-space, cryogenic liquid propellant rocket engines for lunar and Martian exploration missions. Historically, cryogenic rocket engines have not been used for in-space applications due to their complexity, the need for high reliability, and cryogenic propellant fluid management issues. The Apollo Saturn IVB third stage vehicle used a single J-2 LOX-hydrogen rocket engine to reach Earth orbit, for orbit circularization, and for trans-lunar injection. The Apollo service module and the Lunar Excursion Module descent and ascent propulsion used pressure-fed, hypergolic, bi-propellant rocket engines for trajectory adjustment, lunar capture, lunar descent, lunar ascent, and trans-Earth return propulsion. Virtually all planetary exploration missions have used hypergolic propellants for in-space propulsion.

While the mission and vehicle architectures are not defined for the lunar and Mars robotic and human exploration mission objectives, cryogenic rocket engines offer the potential for higher performance and greater architecture/mission flexibility than hypergolic propellants. Recent robotic probes have identified the potential of water ice in the polar regions of the moon and Mars. This discovery allows us to envision mission architectures 
using in-situ propellant production: liquid oxygen on the moon, and liquid oxygen, liquid hydrogen, and liquid methane or other hydrocarbon fuels on Mars. Liquid oxygen can be produced on the moon through thermal decomposition of lunar regolith. Methane can be produced on the Martian surface through chemical reaction of the carbon dioxide atmosphere with hydrogen brought from Earth or with water from Martian regolith or ice deposits. Liquid methane might be the preferred fuel on Mars since it would require less refrigeration to produce and maintain as a cryogenic liquid, and common tankage and liquefaction hardware for storage of both oxygen and methane is possible due to their similar boiling points. Other hydrocarbon fuels such as methanol, ethanol, and hydrocarbon/aromatic blends can also be produced on Mars, but most studies and technology development activities have focused on methane. In-situ propellant production, in combination with in-space propellant storage depots may enable a robust exploration program since it significantly reduces the amount of propellant that must be carried from Earth to space.

Applied technology development is needed if cryogenic liquid propellant rocket engine systems and components are to be included in the exploration program architectures. Recognizing that mission objectives generate mission and vehicle architectures, that in-turn generate engine requirements, for which specific design solutions are created; we see that technology development is directly related to the assumptions used to create the mission concepts and architectures. Stated more concisely, mission architectures dictate the level of technology development required to meet the mission objectives. In order to achieve the exploration objectives, particularly with recognition of a challenging fiscal environment, and ensuring that crew safety is a top priority, the vehicle propulsion systems will be called upon to perform different roles in different missions or mission phases. However, since liquid propellant rocket engines have rarely been designed for multiple uses or applications, the requirements from each of the missions must be examined to determine if a common design can be achieved. The applied technology development goal should be an engine for multiple applications or a family of engines based upon a set of core technologies, design philosophies, and fabrication approaches to reduce overall programmatic cost and risk. The technology challenges can be separated into two groups, first, the system-level challenges resulting from the mission requirements, and second, the subassembly and component-level challenges for implementing the engine system requirements.

\section{MISSION REQUIREMENTS AND BACKGROUND}

By breaking down a planetary exploration mission into its distinct phases, the requirements for each phase can be identified generically and the potential for commonality evaluated. FIGURE 1 identifies the mission phases requiring propulsion systems as Earth-to-Orbit, Trans-Target Injection, Trajectory Correction Maneuvers, Target Capture, Descent-to-Surface, Ascent-to-Orbit, Trans-Earth Injection, Earth Capture, and Earth Reentry. Within the Earth-to-Orbit phase there can be a $1^{\text {st }}$ Stage Boost, a $2^{\text {nd }} / 3^{\text {nd }}$ Stage Ascent, and Orbit Circularization phases. The thrust range for each propulsion system is progressively smaller as the mass being propelled to the required orbital $\Delta \mathrm{V}$ is reduced (recall that the mass returned to Earth during the Apollo missions was $1 / 512^{\text {th }}$ of the initial mass at lift-off).

During the Apollo missions, a common cryogenic LOX/hydrogen engine, the $\mathrm{J}-2$, was used on the $2^{\text {nd }}$ and $3^{\text {rd }}$ stages of the Saturn V launch vehicle for the $2^{\text {nd }}$ and $3^{\text {rd }}$ stage ascent, orbit circularization, and trans-lunar injection (TLI) phases. The J-2 engine had a maximum thrust of $1,000,850 \mathrm{~N}(225,000 \mathrm{lb})$ at an oxidizer to fuel mixture ratio of 5.5:1, however it optimized propellant utilization by reducing the mixture ratio down to 4.5:1 at resultant thrust of $778,440 \mathrm{~N}\left(175,000 \mathrm{lb}_{\mathrm{f}}\right)$. The J-2S version was being developed to increase the mass to LEO capability of the Saturn V launch vehicle and increase mission flexibility. The J-2S had a throttle ratio of 6:1 with a 3\% idle mode. The idle mode simplified the vehicle-engine integration by assisting with cryogenic propellant management. The idle mode was also proposed for low-thrust trajectory maneuvers. The Apollo service module used a bi-propellant, Aerozine $50 / \mathrm{N}_{2} \mathrm{O}_{4}$, engine for trans-lunar trajectory correction maneuvers, lunar orbit capture, and trans-earth insertion propulsion and had a thrust of 95,637 N (21,500 lbf) (Yodzis, 1967). The Apollo lunar descent engine (LMDE) had a throttle ratio of 10:1 to allow terrain avoidance and landing site selection, used the same hypergolic propellants, and had a thrust of 44,482 N (10,000 lbf) (Elverum, 1967). The lunar ascent engine was used only to return the crew from the lunar surface to the service module in lunar orbit. The hypergolic propellants selected for the Service Module and Lunar Excursion Module propulsion was based upon a requirement for guaranteed engine ignition. Not including the $1^{\text {st }}$ stage boost phase, 4 engine development programs were required to meet the mission 
Apollo objectives. Increasing the degree of commonality could provide substantial cost savings to the exploration program.

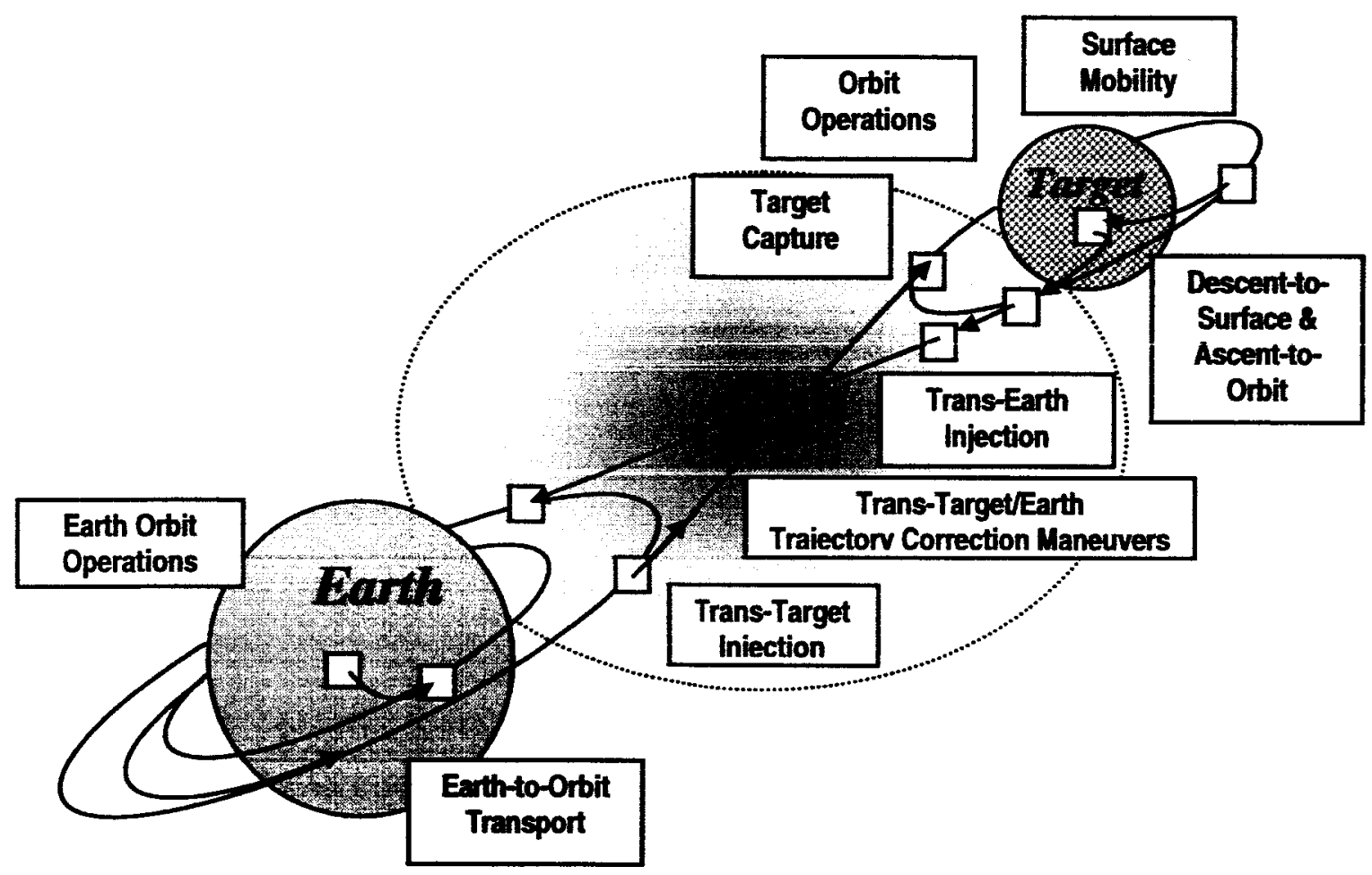

FIGURE 1 - Exploration Mission Phases

A new rocket engine is required because no existing engine can meet the probable requirements from the mission architecture. The only existing domestic in-space cryogenic liquid rocket engine currently in production is the Pratt \& Whitney RL10. The RL10 is an expander cycle, $\mathrm{LOX}_{\mathrm{LH}}$ rocket engine and has had a long, successful history beginning during the Apollo and Centaur programs in the 1960's, to the DCX in the 1990's, to the new Delta IV and Atlas $\mathrm{V}$ expendable launch vehicles now beginning their operational life. The RL10 has evolved over the last 40 years, including configurations with thrust ranging from $66,723 \mathrm{~N}$ to $111,205 \mathrm{~N}(15,000 \mathrm{lbf}$ to $25,000 \mathrm{lbf})$. A throttle capability of 3:1 was demonstrated in the DCX program by replacing the pneumatic actuators with electromechanical actuators. In 1969, a modified RL10 was operated with FLox $\left(\mathrm{O}_{2}-\mathrm{F}_{2}\right)$ and methane, demonstrating expander cycle operation with methane (note, an oxygen-fluorine oxidizer mixture is not desirable for a future system due to flourine's toxicity). The history of the RL10 provides a solid point of departure for development of a future engine and helps guide the technology development program.

Critical to the success of any program is a careful review of lessons learned from past programs and application of appropriate corrective actions to the new program. The lessons learned from existing cryogenic rocket engines, including the RL10 and the Space Shuttle Main Engine (SSME), will be critical to the development of a new engine for exploration missions. While the SSME is very different than the in-space engine being discussed in this paper, a review of major SSME issues identified several fundamental root causes that must be considered as new technologies are developed for the exploration program. The root causes were: 1) inadequate understanding of the engine environment, 2) inadequate systems engineering and integration design trades, 3) inadequate resources, 4) over-estimation of the technology base, 5) immature mission/vehicle design requirements imposed unnecessary engine requirements, 6) inadequate understanding of manufacturing environments and process variability, 7) inadequate understanding of material properties, 8) inadequate quality processes, and 9) high performance requirements (high specific impulse and low weight) drove the design to be very sensitive to all design and operational parameters. 


\section{ENGINE SYSTEM-LEVEL TECHNOLOGY CHALLENGES}

System-level technology challenges are generated from vehicle requirements to meet mission objectives. Multi-use in-space engines are defined as engines that perform for a number of operations to achieve required mission impulses for a variety of trajectory phases, and may operate at different thrust levels during different mission phases. The required throttle range for future lunar or Martian missions is expected to be within our historical experience base, but will depend upon the mission architecture and requirements associated with landing avoidance and plume debris/cratering. The engines must operate after extended space or surface exposure, and inspection and maintenance may not be feasible during its operational life. These requirements affect engine system component design and fabrication. A new lexicon may be needed, our traditional use of the term reusable means the hardware is returned for refurbishment before subsequent operation, but since it is highly unlikely that significant inspection and maintenance can be performed between missions, ithis type of engine should not be classified as "reusable."

Engine system and vehicle stage integration studies must assess the optimum configuration to meet the mission requirements; evaluation items include pressure-fed versus pump-fed engines, engine nozzle design alternatives for vehicle packaging and optimized performance, degree of propulsion system redundancy, and thrust level definition for engine clustering. Studies also need to examine the mass and cost impact of engine optimization for each mission phase versus development of a common engine for multiple mission phase use. For example, can a single engine be used for trans-lunar injection, lunar capture, as well as the descent and ascent phases? Do the requirements for such an engine increase the development cost such that separate systems should be used? Or can clusters of an engine be used for the final ETO impulse, and the same engine be used for the in-space transfer missions, and thus, avoiding the cost of developing different engines for each mission? Trade studies quantifying the ability to combine functions will provide mission architects additional flexibility.

One of the most important trade studies will be the integrated vehicle system trade of a pressure-fed versus pumpfed propulsion system. Historically, in-space systems have been pressure-fed due to their low thrust and short burn duration requirements, requiring small propellant quantities and tanks with acceptable wall thickness (reasonable inert mass). However, if the payload mass or required mission $\Delta \mathrm{V}$ increases significantly, the mass of the pressurefed system may trade unfavorably. Pressure-fed systems have lower engine combustion chamber pressures than pump-fed engines, and for equivalent performance are geometrically much larger. Conversely, pump-fed engines provide a smaller, more efficient system, however the complexity and additional part count can reduce reliability.

\section{Technology Challenges from Vehicle-to-Engine Interface Requirements}

Cryogenic rocket engine system cycle and configuration studies are needed to understand the relative merit of engine system alternatives for the exploration mission architectures. The engine system studies should compare and contrast relevant engine cycles and configuration alternatives by evaluating operation after extended in-space cold soak periods, intra-engine redundancy, options for a multiple restart capability, performance benefits against reliability and complexity, and alternatives for a wide thrust range capability. Recent internal MSFC cryogenic upper-stage engine trade studies are indicating the benefit of expander and tap-off cycles at thrust levels up to 300K, and also identifying configuration alternatives which may provide significant reliability and performance benefits.

A study of engine system and component technology that enables rocket engine operation with LOX/hydrogen propellants during some mission phases and with LOX/methane propellants during other mission phases should be undertaken. This study should determine the feasibility and merits of this approach and determine the technology development required. The study should also investigate other candidate fuels including methanol and ethanol. Even though they are lower performing than methane, applications that are more constrained by volume than mass may prefer to use methanol or ethanol since they are denser and easier to store. An example is a capsule versus a trans-stage.

Since rocket engines have typically been optimized for specific propellants, and since system trade studies have not shown a significant benefit for dual-fuel capability, significant technical challenges may exist in the turbomachinery and combustion devices areas. With appropriate technology and preliminary system development, and with the appropriate mission architecture, a dual-fuel engine could be an enabling technology. A dual-fuel rocket engine presents technical challenges, recalling that the density of liquid hydrogen is $64 \mathrm{~kg} / \mathrm{m}^{3}\left(4 \mathrm{lb} / \mathrm{ft}^{3)}\right.$ and the density of 
cryogenic liquid methane is $424 \mathrm{~kg} / \mathrm{m}^{3}\left(26.5 \mathrm{lb} / \mathrm{ft}^{3)}\right.$, and their associated normal boiling points are $36 \mathrm{R}\left(-423^{\circ} \mathrm{F}\right)$ and $191 \mathrm{R}\left(-268^{\circ} \mathrm{F}\right)$, respectively. The different density, temperature, and heat transfer characteristics between the fuels create significant turbomachinery and combustion design challenges. Such a concept should be given a TRL of 2 at this time. Since the density of methane and ethanol/methanol are much closer than hydrogen and methane, the challenges and degree of difficulty associated with this dual fuel rocket engine concept may be much less.

A multiple restart capability requires advanced ignition and start system development to ensure reliable, robust, and efficient start transients. Applied technology development may be needed in the areas of laser ignition, direct spark ignition, augmented spark ignition, resonance cavity ignition, or other innovative concepts.

In-situ propellant $\left(\mathrm{O}_{2}, \mathrm{H}_{2}\right.$, or $\left.\mathrm{CH}_{4}\right)$ production (ISPP) quality may be less than terrestrial production quality, depending upon the source and production method. The effect of the non-pure propellants must be evaluated with respect to engine performance, safety, and reliability in order to establish a cleanliness tolerance. In-situ propellants may have particulates or other compounds that could affect safety and operation. Current, terrestrial, cryogen production includes residual, trace amounts of inert elements and compounds. Engine testing experience has shown that the nitrogen used for LOX tank pressurization can be absorbed within the oxygen and cause reduced engine performance. Particulates within an oxygen system can be accelerated to high speeds, causing particle impact ignition and catastrophic failure. If liquid oxygen and liquid hydrogen are created by disassociation of the $\mathrm{H}_{2} \mathrm{O}$ molecule, the propellants should be relatively pure. However, if a Sabatier process is used to produce liquid methane from Martian carbon dioxide and regolith water/ice, the potential for contamination is greater. Mars propellant production breadboards that produce just oxygen using the Reverse Water Gas ShifShift (RWGS) process or oxygen and methane using the Sabatier/Water Electrolysis (SWE) process have been built and tested under laboratory conditions, and in one case simulated Mars surface environment, therefore, the TRL is in the 4 to 6 range. The processes used "Earth" supplied hydrogen so the oxygen and methane purity were reasonably "propellant grade." To prevent damage to the water electrolysis unit, water obtained from the moon or Mars will need to be deionized before undergoing electrolysis. Regardless of the ISPP method, it must be thoroughly understood in order to assess the risks associated with propellant contamination.

Engine start and shutdown transients require chill, bleed, purge, and drain systems. These systems must be configured to support the operational mission phases, including any effects on ascent and descent operations. Any propellant or pressurant that is not used to provide mission impulse reduces the efficiency of the vehicle, and since overboard discharges induce forces to the vehicle, innovative technology or design approaches are needed to minimize these effects.

The engine start and shutdown transients must be managed to ensure reliable and safe operation, and for engines that are restarted, the transient conditions must not induce damaging thermal or structural loads. For a lander ascent engine, the transient must be assessed to ensure the rate of thrust build-up is sufficient to maintain vehicle stability. Development of the engine system transient is not a technology development area, the existing tools and capabilities are sufficient for any future program. However, development of a transient analysis tool to support conceptual and preliminary system design could decrease engine development time and more efficiently converge to the optimum system solution.

\section{Engine System-Level Technologies and Analysis Tools}

Once the engine system requirements (performance, packaging, reliability and safety, etc) have been established, based upon the mission objectives and integrated vehicle/engine trade studies, the ability to cost effectively meet those requirements depends upon cross-cutting technologies and analysis tools, primarily within the areas of reliability prediction, high performance materials, and health management.

The engine system thermodynamic cycle (i.e. expander, tap-off, gas generator, staged-combustion) and specific cycle configuration (i.e. full expander, split expander, open or closed expander) chosen for the engine determines the technology investment required to meet the system requirements. Some cycles and cycle configurations currently have a high TRL, but may not support key requirements without technology development, while other configurations may have be at a low TRL, but require less technology development. The throttling requirement and the degree of control needed may necessitate certain system configuration and engine component design approaches for the valves, injector, combustion chamber, and turbopump. For example, the linear, proportional throttle response 
of the LMDE was a result of the integrated valve and injector design approach. However, modern control system and valve technologies may allow other injector concepts and yield a more optimum system solution. A system solution to provide a very wide throttle range, with low technology and development risk, may be obtained by operating in a pressure-fed mode at low thrust, "idle" mode, and in pump-fed mode at high thrust, mainstage. Such solutions that trade technology development against clever system solutions must be thoroughly investigated and are only possible by developing and assessing multiple conceptual designs at sufficient fidelity.

High system and component reliability is required to provide mission success. A capability gap exists in the design and analysis tools and methodologies to ensure that reliability is designed into the system and components, and not simply considered as an assessed attribute. The tools for incorporating physics-based reliability into the design process have not been developed for liquid propellant rocket engine systems, as well as how to design for humanrating requirements and the appropriate level of redundancy. Design and analysis tool technology development is needed in several areas to meet the assumed long-life, high reliability system requirements, including engine system structural dynamics, fracture mechanics and crack propagation, component performance uncertainty analysis, and other probabilistic design methods.

Materials with higher specific strength at higher operating temperatures with enhanced compatibility in the natural and induced environments will increase the capability of the engine components. Methane chemical compatibility in thrust chamber cooling environment must be evaluated, studies conducted in the 1980's indicated issues with some coolant liner materials, particularly when the methane contained sulfur contaminants. Material environmental compatibility must be assessed to determine if the environmental conditions the engine is exposed to during the mission or pre-mission acceptance testing can damage the engine hardware and decrease reliability. Environmental exposure includes surface soil, dust, atomic elements, radiation, and micrometeorites. For hydrogen engines, hydrogen embrittlement resistant materials are required, such as MSFC developed NASA-23. A significant engine development cost and schedule issue is the availability of material property data at the beginning of the design and analysis phase. If sufficient material test data is not available, the programmatic risk may be too great to integrate it into the design before extensive testing is complete, and this often leads to not utilizing a superior material. The same logic applies to component fabrication processes, if process has not been sufficiently matured, a project manager may chose a more conventional process to avoid the technical risk. The following materials and fabrication processes have shown potential for increasing the mass efficiency of the system: GRCop-84, NASA-23, powder metallurgy with vacumm plasma spray (VPS) or selective net shape hot isostatic press (HIP),

Engine system health management system technology development is needed in the areas of condition monitoring and system assessment to support decision algorithms. The engine must have the ability to monitor its own health and determine whether a detected anomaly will result in a system failure condition, and if so, can the engine be operated in a more benign condition to mitigate the failure, or can the engine be shutdown (if the system was designed with multiple engines to allow engine-out operation). The long durations of expected exploration missions would benefit from an engine health management system through an ability to detect anomalous engine performance, assess the probability of failure, and recommend corrective action autonomously. An example would be the detection of an abnormal turbopump vibration and recommendation to throttle the engine to a lower power level and a more benign environment. Another example would be the detection of a propellant leak and recommendation to change the engine power level or mixture ratio to maintain desired propellant utilization.

\section{SUBASSEMBLY AND COMPONENT TECHNOLOGY CHALLENGES}

The mission requirements which affect the detailed component designs include throttle ratio, environment and operational duty cycle, performance, packaging, pre-start and shutdown conditions, reliability, safety, and, most significantly, the propellant selection. In some form or another, those requirements affect all of the engine component designs. However, some are much more critical than others. For example, a deep throttle requirement significantly affects the injector, thrust chamber, turbomachinery, and valve design. In addition, service life requirements for heritage reusable engines have typically been on the order of 30 cycles, while multi-use, multistart, in-space applications will require technology advances to significantly increase service life capabilities and improve reliability. Achieving substantial weight reduction will also be required to enable lower risk architectures, by ultimately requiring smaller launch vehicles. These factors all dictate a need for improvement in engine components. 


\section{Combustion Devices}

Applied technology development for the design of injectors, combustion chambers, and high area ratio nozzles is needed to meet the life and operational mission requirements. Injector and combustion chamber technology development areas are contingent upon the engine system cycle selected to meet the mission requirements. Innovative and evolutionary nozzle design approaches to satisfy vehicle integration and mission requirements is needed. Potential concepts could include extending nozzles during operation, expansion-deflection nozzles, and dual-bell nozzles. Although, cryogenic rocket engines have typically not needed a large throttle capability, it will be necessary to achieve multiple-use and multiple-mission applications. High throttle ratio capability requires applied technology development in injector design to provide stable combustion over the entire operating range. Throttling reduces the propellant mass flowrate through the engine, causing reduced pressure drop through the injector and coupling of fluid dynamic and combustion processes leading to unstable combustion. In the worst case, undamped combustion instabilities can result in catastrophic failures. The most significant thrust chamber challenge is balancing system performance with the thermal capability of coolant liners at deep-throttle, low coolant flow operation. Since hydrocarbon fuels are more prone to combustion instabilities, the evaluation of LOX/methane combustion stability performance at throttled conditions is a specific area of concern. Advanced materials and fabrication processes are needed for combustion chambers and nozzles. The high operational pressures of thrust chamber coolant circuits and thrust related stresses typically require high strength super alloys for load reaction, ultimately a major engine weight driver. High strength enhanced thermal conductivity liner material and high strength structural alloy technology maturation is needed to address the design objectives.

However, the most significant technology gap for a multiple restart capability is ensuring a reliable, robust, efficient start transient. In particular, an advanced ignition and start system development is needed to provide cryogenic engines with start reliability similar to hypergolic engines. The advantage of hypergolic propellants is that one the propellants mix in the main combustion chamber, they react and burn spontaneously. To achieve that degree of robust combustion chamber ignition, technology development efforts should be pursued. Laser, spark torch, direct spark, resonance cavity, and combustion wave ignition concepts may all have potential applications.

\section{Turbomachinery}

High throttle ratio capability requires turbomachinery technology development to obtain stable operation across a wide range of flow conditions. Since the turbomachinery is also operating significantly away from the design points during throttled operation, negative effects can include pump stall, flow instabilities, and rotating cavitation. Rotating cavitation occurs when the propellant speed across the rotating impeller blade is such that the local pressure is below the propellant vapor point and the propellant vaporizes locally. The formation and collapse of the vapor bubbles causes erosion of the blade, leading to additional friction, lowered performance, and the potential for structural failure. The advances in computational fluid dynamics, coupled with and anchored against flow tests, has made great advances in these areas, however these tools need continued technology investment to avoid design iterations during system development. Cryogenic rocket engines are sensitive to propellant inlet conditions. Pumps prefer to start at higher inlet pressures and colder temperatures, however in-space vehicle stages prefers lower pressure and higher temperatures to reduce propellant tank mass. Technology development is needed to enable robust engine starts at low pump net positive suction pressures (NPSP), i.e. low pressure and high temperature propellants. This technology is integrally related to the in-space cryogenic fluid management technologies.

\section{Valves and Actuators}

Considerable development is required for valves and actuators, since the anticipated requirements for this application are unique and outside the capabilities of existing valves. The mission expected duration necessitates very low leakage requirements. Existing valves for long duration missions typically provide lower flow rates and lower operating pressures than those expected for this application. The throttle requirement for this engine will call for control valves, while valves used currently for long duration missions are typically on/off latching valves. Valve reliability and redundancy requirements will be unique if the engine will operate in both descent and ascent mission phases. The multiple restart capability, with the possibility of extended periods of in-space cold-soak, creates unique environment conditions that previous cryogenic rocket engine valves and actuators have not had to endure. 
Electric based actuators may provide a system-level benefit by reducing the quantity of on-board consumables (helium or nitrogen gas), and while electro-mechanical actuators have extensive experience on aircraft, the safety and reliability requirements for exploration missions requires improvements. An axiom of old rocket engineers is that valves leak. However the anticipated mission requirements cannot tolerant valve leakage and the loss of propellants, therefore innovative design approaches must be found. The throttle requirements for planetary lander spacecraft are implemented by the propellant valves. For the Apollo LMDE, the valves and injector were an integral unit, and included a set of variable area cavitating venturi valves to provide linear, predictable engine thrust control. Such a design approach may or may not be compatible with the engine system design. But since accuracy and controllability will be prime considerations; development of alternative valve and actuator technologies is needed.

\section{Avionics and Instrumentation}

Avionics and instrumentation system have been the component with the highest rate of removal for cause on the SSME. Without control system integrity, including robust sensors that can operate accurately over extended periods in space, the reliability of the entire propulsion system is questionable. The degree of avionic system redundancy must be addressed, including hardware and software technology development to make development and integration easier. Increased operational safety and reliability goals has led to development of Engine Health Management Systems (EHMS) to continually assess engine system and component health. EHMS development is needed in the areas of instrumentation and sensor systems, algorithm development, and hardware/software integration to achieve reliability, safety, operability, and maintainability goals. Technologies such as advanced data busses and alternate protocols, multi-element sensors, leak detection sensors, smart sensors and actuators are but a few examples.

\section{CONCLUSION}

The development of advanced rocket engine systems to meet the objectives of the President's Exploration Vision is within the current or near-term state-of-the-art for rocket propulsion systems, using a focused technology program to address current shortfalls and extrapolation of technology to different usage. Following a traditional Systems Engineering approach, after the architecture capabilities are defined to achieve the desired mission objectives, the requirements for the vehicle subsystems can be developed. It is impossible to fully define the propulsion system technology needs in the absense of well-developed mission and vehicle architecture concept development; however, the Apollo system capabilities and existing architecture concept studies can be used to identify likely requirements. This synthesized set of requirements can then be used to define the engine system and configuration concepts based upon specific component capabilities. The component and analysis required capabilities thus identified can then be used to identify specific technologies for maturation. The zeroth-order analysis presented in the paper identifies the areas within combustion chamber, injector, turbomachinery, valves, and actuators as needing focused predevelopment activity to enable future missions. This type of pre-development will help the final mission architecutre definition by avoiding an over-estimation of the technology base, leading to expensive test-fail-fix cycles during system development. The driving requirement for a deep-throttle multi-use in-space rocket engine is mission reliability. While much of that reliability may be able to be met through the architecture design, using multiple engines to allow an engine-out capability as an example, developing specific engine system and component design approaches to ensure robust, reliable operation requires near-term technology investment. Technology areas associated with reliable engine starts, stable, predictable throttling, and efficient shutdowns that allow for engine restart after extended coast or on-surface periods should be highest priorities.

\section{REFERENCES}

Elverum, G. Jr., Staudhammer, P., Miller, J., Hoffman, and Rockow, R., "The Descent Engine for the Lunar Module," AIAA $3^{\text {rd }}$ Propulsion Joint Specialist Conference, Washington, D.C., AIAA Paper No. 67-521, 1967.

Yodzis, Charles W., "Engines for Manned Spacecraft," AIAA $4^{\text {th }}$ Propulsion Joint Specialist Conference, Cleveland, OH, AIAA Paper No. 68-567, 1968. 